\title{
Cytogenetic karyotype analysis in selected species of the Erinaceidae family
}

\author{
Anna Grzesiakowska ${ }^{1}$, Przemysław Baran ${ }^{2}$, \\ Marta Kuchta-Gładysz ${ }^{1}$, Olga Szeleszczuk ${ }^{1}$ \\ ${ }^{1}$ Department of Animal Anatomy, Institute of Veterinary Sciences, \\ University of Agriculture in Kraków, 30-059 Kraków, Poland \\ ${ }^{2}$ Salamandra Veterinary Clinic in Kraków, 30-052 Kraków, Poland \\ grzesiakowska.anna.ur@gmail.com
}

Received: December 21, 2019 Accepted: July 2, 2019

\begin{abstract}
Introduction: Comparative analysis of the karyotype structure was made in two hedgehog species: the northern whitebreasted hedgehog (Erinaceus roumanicus) and the African pygmy hedgehog (Atelerix albiventris). Material and Methods: The cytogenetic analysis used differential staining techniques (DAPI, Ag-NOR, and C-banding/DAPI) and sequential QFQ/FISH banding with NOR20 and TEL20 probes which showed 45S rDNA and (TTAGGG) $)_{n}$ repeat sequences, respectively, on hedgehog chromosomes. Results: It was confirmed that the somatic cells of the hedgehogs have a constant number of chromosomes $(2 \mathrm{n}=48, \mathrm{XY})$. Differences were observed in the NOR number between the species. NORs were identified on three autosome pairs in the northern white-breasted hedgehog and on only two pairs in the African pygmy hedgehog. Chromosome analysis by C-banding/DAPI showed large segments of heterochromatin rich in A-T pairs on three autosome pairs in both the northern whitebreasted and African pygmy hedgehogs. The heterochromatin segments encompassed large fragments of the longer arm of chromosome pairs 13, 14 and 20. The (TTAGGG) repeat sequences on the hedgehog chromosomes were only observed in the terminal position of all the chromosomes in both species. Conclusion: Our observations provide new information on the level of diversity within the Erinaceidae family.
\end{abstract}

Keywords: hedgehog, chromosomes, heterochromatin, nucleolar organisers, telomere.

\section{Introduction}

The interest in and research on the cytogenetic characteristics of hedgehog karyotypes date back to between the late 1960s and the 1980s $(7,10,13)$. These studies concentrated on identifying individual karyotype traits, mainly in the northern white-breasted hedgehog as the species inhabiting Europe $(12,13)$ and native species found in different territories $(2,5,14)$.

Hedgehogs are members of the subfamily Erinaceinae in the order Erinaceomorpha. They have a wide territorial range, different regions inhabited by different species and subspecies. The northern whitebreasted hedgehog comprises two species. The first is Erinaceus europaeus, known as the western European hedgehog. It is found mainly in northern Europe and Scandinavia, but also in central Asia (1). The second species is the eastern European hedgehog (Erinaceus roumanicus) (1), known as the northern white-breasted hedgehog. It inhabits central and eastern Europe (Poland, Austria, Ukraine, and Russia), the peninsulas of the Baltic Sea, the Balkans, and north-eastern Turkey (3). Taxonomically, it was originally recognised as the subspecies E. europaeus and later as the subspecies E. concolor. Today, E. roumanicus constitutes a separate species, and along with E. concolor (Martin 1838), it is grouped among the eastern European (white-breasted) hedgehogs (1). Mandahl (13) was the first to designate E. roumanicus as an eastern European species. The second species of the eastern European hedgehog group, E. concolor or southern white-breasted hedgehog, is distributed in Asia Minor and the Mediterranean region. The karyotype of the northern white-breasted hedgehog (E. roumanicus) has a diploid chromosome number of $2 \mathrm{n}=48$. The chromosome complement includes, within autosomes, 12 pairs of metacentric chromosomes (M), six pairs of submetacentric chromosomes (SM), four 
pairs of subtelocentric chromosomes (ST), and one pair of acrocentric chromosomes (A). The metacentric sex chromosomes are made up of a medium-sized $\mathrm{X}$ chromosome and a small Y chromosome (1).

A separate group is formed by the Atelerix species, which also belong to the Erinaceidae family. This group is represented by Atelerix albiventris, known as the African pygmy or four-toed hedgehog. It occurs naturally in eastern and northern Africa and in Spain and France (9). Currently, African pygmy hedgehogs are found in various areas. Due to the growing popularity of keeping African pygmy hedgehogs as pets, their territorial range is gradually coinciding with that of the northern white-breasted hedgehog. The karyotype of the African pygmy hedgehog is $2 \mathrm{n}=48$. Morphologically, within autosomes, 1 large pair of SM and M chromosomes, 18 pairs of medium-sized SM and $\mathrm{M}$ chromosomes, and 3 pairs of small chromosomes (including one $\mathrm{M}$ and two SM) have been described. The sex chromosomes include a large $\mathrm{X}$ chromosome and a small $\mathrm{Y}$ chromosome, both of the metacentric type (9).

To date, cytogenetic studies in hedgehogs have concentrated on characterising the karyotypes of different species, in particular the European hedgehogs $(6,12,13)$. There are no studies addressing other species or the differences in chromosomal structure among Erinaceus roumanicus, Erinaceus europaeus, and Atelerix albiventris. The available literature data concerning karyotype studies mainly include $\mathrm{G}$ and $\mathrm{C}$ banding techniques (12) as well as Ag-NOR staining (13) in eastern and western European hedgehogs. The same staining techniques were used by Hübner et al. (9) to study the karyotype of the African pygmy hedgehog and by Arslan et al. (1) to determine the karyotype and the differences in heterochromatin distribution between two species of hedgehogs found in Turkey. Sanchez et al. (14), who karyotyped the Algerian hedgehog (Erinaceus algirus) from northern Morocco, complemented G, C, and Ag-NOR banding with in situ hybridisation with an rDNA probe for additional analysis of the location of nucleolar organiser regions (NORs).

The aim of the study was to perform comparative analysis of the karyotypes of the two hedgehog species Erinaceus roumanicus and Atelerix albiventris, with special consideration of the diploid chromosome number, the number and sites of NORs in the chromosome complement, and the location of (TTAGGG) $)_{\mathrm{n}}$ repeat sequences on the chromosomes of both species.

\section{Material and Methods}

Material. The study was performed with six hedgehogs representing two different species: the northern white-breasted hedgehog (Erinaceus roumanicus) and the African pygmy hedgehog
(Atelerix albiventris). For each species, the experimental group was made up of three breeding males of the same age (one year old). The animals originated from the population of hedgehogs found in the area of Kraków, Poland. The material of the study was whole peripheral blood collected during routine veterinary tests with the owner's consent.

Chromosome preparations. The study used metaphase chromosomes obtained from in vitro culture of whole peripheral blood lymphocytes. The cell cultures were carried out in LymphoGrow medium (Cytogen, Poland) under standard conditions $(72 \mathrm{~h}$, $\mathrm{T} 37.5^{\circ} \mathrm{C}, 5 \% \mathrm{CO}$, with constant humidity).

Chromosome staining methods. To determine the number and general morphology of the chromosomes, the preparations were stained with a mixture of DAPI (4',6-diamidino-2-phenylindole) and Vectashield (Sigma-Aldrich, Germany). The karyotype of both hedgehog species was subjected to C-banding/DAPI procedure to show the segments of heterochromatin enriched in A-T nitrogen base pairs (15). The number and sites of active NORs in the chromosome complement were identified by means of Ag-NOR staining (8).

Fluorescence in situ hybridisation (FISH). In the sequential analysis, the QFQ/FISH technique was used, and the chromosomes were identified with QFQ differential staining (4). The (TTAGGG) $)_{n}$ repeat sequence was located on chromosomes using FISH with a telomeric probe (TEL20, Chrombios, Germany) following the manufacturer's protocol. The signals of 45S rRNA loci were likewise located on chromosomes using FISH with a NOR20 probe (Chrombios) following the manufacturer's protocol.

Analysis of chromosome preparations. The microscopic analysis of the preparations and the photographic documentation of the chromosomes were made under an Imager A2 epifluorescence microscope fitted with an AxioCam MRc 5 digital camera, (Zeiss, Germany). The metaphase plates were analysed with the image analysis software NIS-Elements F 2.31 (Nikon, Japan) and MultiScan Base 6.08 (MultiScan, Poland).

\section{Results}

A total of 100 metaphases were analysed for each animal. DAPI analysis of the preparations confirmed that all the animals had a constant diploid chromosome number of $2 \mathrm{n}=48, \mathrm{XY}$ (Figs $1 \mathrm{a}$ and $\mathrm{b}$ ).

Further analysis consisted in evaluation of the karyotype morphology of both hedgehog species and the performance of the QFQ technique used. The staining showed that the fundamental arm number (NF) in $E$. roumanicus was 94 and the autosomal arm number (NFa) was 90. Four morphological types of chromosomes were observed in the karyotype within the autosome group. For the metacentric chromosomes, 
these were pairs 1-11 and autosome pair 22 (12 pairs in total). The submetacentric chromosome type was represented by six pairs (autosome pairs 15-19 and 23), and the subtelocentric type chromosomes were present as four pairs (pairs 12-14 and 20) and one acrocentric pair (no. 21). The karyotype analysis of $A$. albiventris showed a NF of 96 and a NFa of 92. Fourteen pairs of metacentric $(\mathrm{M})$ autosomes were described (pairs 1, 3-5, 7, 10, 12-16, and 18-20). Within the submetacentric type (SM), six autosome pairs $(2,6,8-9,11$, and 17) were identified. Also three pairs of small chromosomes, including one of the metacentric type (no. 21) and two of the submetacentric type (nos. 22 and 23) were identified. In total, 15 pairs of
M chromosomes and 8 pairs of SM chromosomes were observed in the A. albriventris karyotype within the autosomes. For both species, there were no differences in the sex chromosome morphology, where $\mathrm{X}$ and $\mathrm{Y}$ chromosomes were of the metacentric type and the $\mathrm{Y}$ chromosome was in both cases one of the smaller metacentric chromosomes in the karyotype. The karyotype analysis demonstrated differences in the morphology of autosome pair 21 (Figs 1c and d). In the case of the European hedgehogs, these were small acrocentric chromosomes. In African pygmy hedgehogs, chromosome pair 21 was formed by submetacentric type chromosomes.

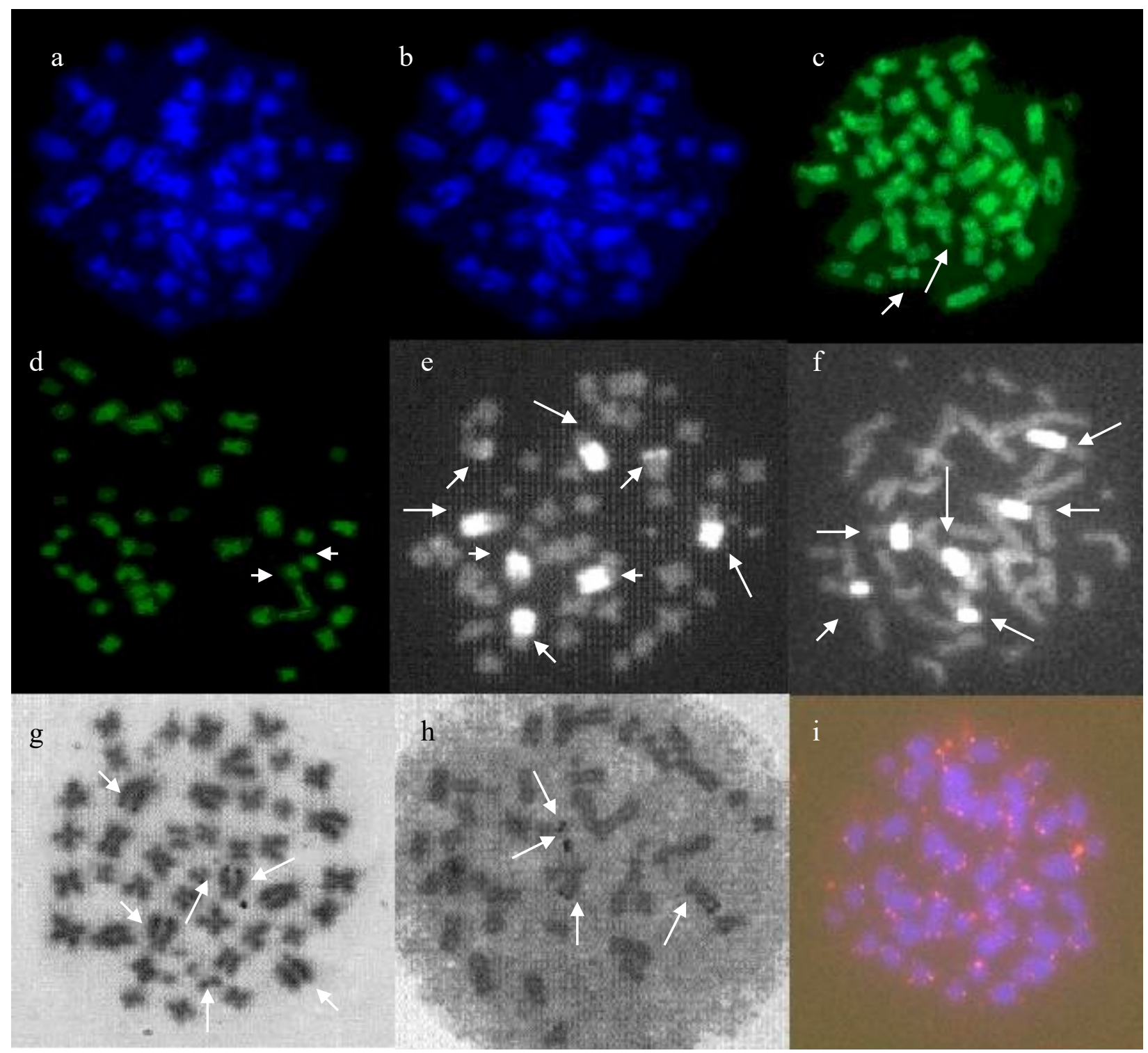

Fig. 1. Chromosomes of hedgehogs in various cytogenetic techniques

a - DAPI-stained chromosomes of Erinaceus roumanicus; b - DAPI-stained chromosomes of Atelerix albiventris; c - QFQ-stained metaphase plates of Erinaceus roumanicus. Arrows indicate chromosome pair 21; d-QFQ-stained metaphase plates of Atelerix albiventris. Arrows indicate chromosome pair 21; e - C-banding/DAPI-stained metaphase plates, $2 \mathrm{n}=48$ chromosomes of Erinaceus roumanicus. Arrows indicate chromosomes with segments of A-T-rich heterochromatin; f - C-banding/DAPI-stained metaphase plates, $2 \mathrm{n}=48$ chromosomes of Atelerix albiventris. Arrows indicate chromosomes with segments of A-T-rich heterochromatin; g - Ag-NOR-stained chromosomes of Erinaceus roumanicus. Arrows indicate chromosomes with NORs; h - Ag-NOR-stained chromosomes of Atelerix albiventris. Arrows indicate chromosomes with NORs; i - metaphase plates stained by FISH with TEL20 probe in E. roumanicus karyotype. Scale bar $10 \mu \mathrm{m}$ 
In comparing the hedgehog species from the area of Kraków, distribution of A-T rich constitutive heterochromatin was used. Heterochromatin blocks were observed as bright spots on chromosomes (Figs 1e and $\mathrm{f}$ ). In the case of the European hedgehogs, large heterochromatin blocks were observed in three chromosome pairs (Fig. 1e). These bands occurred on autosome pairs 13 and 14 in all the animals under study. The third heterochromatin block was observed on autosome pair 20 in most of the animals. In a few samples, there were differences in distribution of the heterochromatin block, which was observed on autosome pair 21. This may be indicative of interspecific differences between hedgehogs from the studied area. In each case, the AT-rich segments occurred on the $\mathrm{q}$ arms and covered the whole area $(13 q, 14 q, 20 q$, or 21q). In the case of the European hedgehogs, an additional heterochromatin segment was found in the terminal part of the $\mathrm{q}$ arm, namely on chromosome pair 15 (15q) (Fig. 1e). For the African pygmy hedgehogs, heterochromatin blocks were observed on the $\mathrm{q}$ arms of autosome pairs 13, 14, and 20 (Fig. 1f).

The number and location of active NORs on the chromosomes were evaluated in both hedgehog species using Ag-NOR banding. In the case of the northern white-breasted hedgehog, NORs were localised in the distal part of the $\mathrm{q}$ arm on chromosome pairs 13, 14, and 20 (Fig. 1g). In the African pygmy hedgehog cells, active NORs were located on two pairs of autosomes 13 and 21 and in the distal part of the long $\mathrm{q}$ arm (Fig. 1h). FISH with a NOR20 probe was additionally used. Probe signals for the 45S rRNA loci were identified on chromosome pairs 13, 14, and 20 in E. roumanicus, which coincides with the distribution of active NORs (Ag-NOR bands). A similar characteristic was shown by the A. albiventris karyotype, in which the localisation of inactive NORs coincided with the distribution of NORs.

Based on FISH with the human telomere sequence (5'TTAGGG3'), telomeric sequences were only observed at the end of all chromosome arms (Fig. 1i).

\section{Discussion}

The family Erinaceidae contains various types of small mammals, including five genera of hedgehogs: Erinaceus, Atelerix, Hemiechinus, Mesechinus, and Paraechinus (9). The individual species have a very wide distribution area. The present study focused on cytogenetic characteristics and karyotype comparison of the members of the first two genera, namely Erinaceus roumanicus and Atelerix albiventris.

The karyotype analysis confirmed the presence of the same diploid number of chromosomes $(2 n=48)$ in the cells of both species. In the northern white-breasted hedgehog, the morphological evaluation performed according to the criteria proposed by Levan et al. (11) showed the presence of all four chromosome types in the karyotype. The most numerous were metacentric chromosomes, 12 autosome pairs, and $\mathrm{X}$ and $\mathrm{Y}$ chromosomes. The submetacentric type was identified on six autosome pairs and the subtelocentric type on four chromosome pairs. The last morphological type of chromosomes (acrocentric) was only found for one autosome pair (no. 21). The present characteristics of the E. roumanicus karyotype match the observations of Arslan et al. (1). In his study of the karyotypes of European hedgehogs, Mandahl (12) distinguished five different karyotypes, three belonging to the western hedgehogs and two to the eastern ones (EI and EII). In two karyotype variants for the eastern hedgehogs, he observed 13 pairs of metacentric chromosomes (11 pairs of large and medium-sized chromosomes as well as 2 pairs of very small chromosomes). Sex chromosomes were also identified as metacentric. He divided the other 10 autosome pairs into three groups, the smallest of which was pair 21 , designated as the telocentric or acrocentric type. Mandahl (12) further classified the next nine autosome pairs as subtelocentric (four pairs) and submetacentric chromosomes (five pairs). For both karyotype variants (EI and EII), these chromosome pairs shared the same characteristics. Based on the analysed metaphase plates, it was found that the observed karyotype of European hedgehogs was closer to the EII karyotype variant proposed by Mandahl (12).

Based on the observations of DAPI- and QFQstained metaphase plates in the material obtained from the European hedgehogs, differences were found in size in acrocentric chromosome pair 21. These differences were observed only in very few cells. In most of the material, pair 21 was easily identified as small acrocentric chromosomes because they formed the third pair of chromosomes which were very small compared to the other chromosome pairs in the karyotype. In some of the samples, the acrocentric chromosome pair was larger and its size was close to that of submetacentric chromosomes. These observations led us to conclude that the cells with larger acrocentric chromosomes probably originate from an animal with a different karyotype variant. Referring to the description of different karyotype variants identified by Mandahl (12), we found that the additional karyotype observed in part of the material points to the western European hedgehog (Erinaceus europaeus). The most significant karyotype difference between the western European and northern white-breasted hedgehogs (E. roumanicus) is the size of acrocentric pair 21, which is of medium size in the former and forms small chromosomes in the latter $(12,13)$.

Besides Erinaceus roumanicus, the northern white-breasted hedgehog also includes E. concolor. The available literature data were used to compare the E. concolor karyotype with the material analysed as part of the present study. No differences were found in the number or morphological type of chromosomes 
between these species. The observations agree with Arslan et al. (1) who compared both species sampled from Turkey. Sanchez et al. (14), who investigated the karyotype of the Algerian hedgehog (Erinaceus algirus) from Morocco, observed the same diploid chromosome number of $2 \mathrm{n}=48$ and a similar distribution of the chromosome pairs of different morphological types. The observed differences in the karyotype of the European hedgehog concerned the size of chromosome pairs 14 and 21. The chromosome pair 14 observed by Sanchez et al. (14) was subtelocentric and medium in size, while pair 21 was small and acrocentric, which matches the size and type of these chromosome pairs in E. roumanicus.

Although the African pygmy hedgehog (Atelerix albiventris) belongs to a different genus, it has the same diploid number of chromosomes as the Erinaceus species. In both African pygmy hedgehogs and those of the Erinaceus genus, metacentric chromosomes are most common in the karyotype. The other chromosomes were submetacentric. In A. albiventris, no acrocentric or subtelocentric chromosomes were found, which distinguishes this species from the European hedgehogs. Similar observations of the types and morphological classification of chromosomes in A. albiventris were reported by Hübner et al. (9).

Karyotypes contain different repeat sequences, which may be indicative of taxonomic relatedness or interspecies differences. One example of such sequences are nucleolar organiser regions, which were visualised by Ag-NOR staining. In the present study, we identified NOR signals in the distal parts of the long arms of six chromosomes (pairs 13, 14, and 20) for E. roumanicus. Mandahl (13) determined the mean number of active NORs to be six (in a range of five to eight), which occur distally in both European hedgehog species on three to five autosome pairs. Irrespective of the European hedgehog species, Mandahl (13) identified NORs on the long arms in the distal part of chromosomes 13, 14, 15, 19, and 20. For pair 21, the localisation of NORs varied according to the species on the short arm in E. roumanicus and on the long arm in E. europaeus. In E. roumanicus from five different areas of Turkey, Arslan et al. (1) identified active NORs in the terminal regions of the long arm on four autosome pairs $(13,14,15$, and 20). The position of these NORs corresponded with that described by Mandahl (13) for animals with the EII karyotype from Poland (Kraków). These observations agree with our observations of active NORs in E. roumanicus from the area of Kraków. In some of the animals from northeastern Turkey, NORs were found distally on the q arm of autosome pairs 13,14, and 19 and on the short arm of pair 21 (1). In E. concolor from Turkey, there were four pairs of active NORs on autosome pairs 13 and 14, and, depending on distribution region, on pairs 17 and 21 , or 15 and 20 (1). In the case of E. algirus, NORs were identified interstitially on chromosome pairs 13, 21, and 23, and distally on the long arm of pairs 15 and 16
(14). The African pygmy hedgehogs (A. albiventris) were characterised by only two pairs of active NORs, which were located distally on chromosome pairs 13 and 21 .

The present study also concentrated on analysing the occurrence and distribution of heterochromatin segments using C-banding/DAPI staining. This technique enables observation of the distribution of heterochromatin segments rich in AT pairs. The staining performed in the two analysed species $E$. roumanicus and $A$. albiventris revealed the presence of large heterochromatin blocks occupying large fragments of the long arm of the same chromosome pairs 13, 14, and 20. In the European hedgehog, there was an additional heterochromatin band in the terminal part of the $\mathrm{q}$ arm of chromosome 15. In the African pygmy hedgehog (A. albiventris) after C-banding with barium hydroxide and Giemsa, Hübner et al. (9) did not observe any positive heterochromatic material. Geiser and Gropp (6) considered the distribution of heterochromatin blocks as the main factor differentiating the karyotypes of European hedgehogs. In the study by Arslan et al. (1), C-banding revealed no bands near the centromere in any of the specimens, which was confirmed in the present study. The most extensive analysis of C-blocks in the European hedgehog karyotypes was performed by Mandahl (12), who described their polymorphism and considered them a source of interspecific variation. In his study, Mandahl (12) observed that on the chromosomes of the European hedgehogs, C-positive regions form large blocks which are mostly located distally on two to four autosome pairs. Our observations showed the presence of large heterochromatin blocks on three autosome pairs. When describing in detail differences in the distribution of heterochromatin between different karyotype variants of western European and northern white-breasted hedgehogs, Mandahl (12) found large variation for all variants in these species. He noted that the greatest difference in the localisation of C-blocks concerns chromosome pairs 20 and 21. In the E. roumanicus karyotype, the C-block is found on chromosome pair 20 but is missing from autosome pair 21. An inverse distribution of this block occurs in E. europaenus, where it is found on chromosome pair 21 but not on 20 (12). The present study confirms observations made by Mandahl (12) and Arslan et al. (1) concerning the presence of an additional small heterochromatin block on autosome pair 15 in the northern white-breasted hedgehog. The observations concerning the distribution of C-bands on autosome pairs made in this study verified research conducted by Arslan et al. (1) for E. concolor originating from six different areas of Turkey, who found them to occur on autosome pairs 13, 14, and 20. In the case of E. roumanicus, they located the heterochromatin on chromosomes 13, 14, 15, and 20 (1). In a study on heterochromatin distribution in E. concolor from Romania, Gavrila et al. (5) observed that constitutive 
heterochromatin bands covered two-thirds of the length of chromosomes on three autosome pairs. The distribution of C-blocks presented by Gavrila et al. (5) in E. concolor differs from that of the E. roumanicus or E. europaeus karyotype. In E. concolor, heterochromatin was observed on the q arm of chromosome pairs 1-3 in addition to there being small C-bands on chromosome 18 in the telomeric region and on chromosome 19 in the centromeric region (5). In turn, the presence of two large C-blocks in the distal region of chromosome pairs 13 and 14 in E. algirus was confirmed by Sanchez et al. (14). Research in different Erinaceus species confirmed that they are characterised by the presence of large C-blocks, most often on autosomes 13 and 14 . Other observations of the heterochromatin distribution were made by Arslan et al. (2) for Hemechinus hedgehogs. The Hemechinus auritus karyotype was characterised by distinct centromere heterochromatin blocks on seven autosome pairs (4, 6-9, 12, and 17), while pair 21 consisted entirely of heterochromatin (2).

Studies with different hedgehog species and with specimens from different geographical areas showed that the heterochromatin and NOR are related in two chromosome pairs (13 and 14$)$ in E. roumanicus, E. europaeus, and E. concolor $(12,13,1)$ and within pair 13 in E. algirus (14) and A. albiventris, which may be indicative of a high relatedness cytotaxonomically.

The available literature data provide no information about using FISH in analysis of repeat sequences. Another type of repeat sequences are telomeric sequences, which were analysed using a 5'TTAGGG3' telomere probe. They were found to occur only at the end of all chromosome arms in both hedgehog species under study.

It seems important to identify exact differences and similarities in the karyotypes of European and African pygmy hedgehogs due to constant changes in the distribution area of these species. This does not only involve changes that may occur naturally as a result of the interbreeding of the northern whitebreasted and western European hedgehog species pair. It seems that interspecies crosses will be created naturally as a result of the overlapping habitats of these two species. Bogdanov et al. (3), who investigated the karyotype of E. europaeus and E. roumanicus interspecies crosses, found a greater morphological and C-block similarity for E roumanicus. The presence of NOR signals mostly matched the NOR pairs for E. roumanicus (3). This research suggests that the crossing process may be taking place. Considering the growing interest in keeping African pygmy hedgehogs as pets, this may increase genetic variation of hedgehogs and lead to the generation of new interspecific crosses.

Conflict of Interests Statement: The authors declare that there is no conflict of interests regarding the publication of this article.
Financial Disclosure Statement: The study was supported by the Mission-Related Research project DS-3252/ZAZ of the University of Agriculture in Kraków.

Animal Rights Statement: The research material was collected during veterinary research aimed at prevention of animal diseases and their diagnosis according to the Act of 15 January 2015.

\section{References}

1. Arslan A., Zima J., Özparlak H.: C-heterochromatin variation in the karyotype reflects species level distinction between Erinaceus roumanicus and E. concolor (Eulipotyphla: Erinaceidae) in Turkey. Zootaxa 2008, 1961, 1-10.

2. Arslan A., Albayrak I., Pamukoğlu N., Yorulmaz T., Toyran K.: C-banded karyotype and NORs of the long-eared hedgehog, Hemiechinus auritus from Turkey. Folia Zool 2009, 58, 9-13.

3. Bogdanov A.S., Bannikova A.A., Pirusskii Yu.M., Formozov N.A.: The first genetic evidence of hybridization between west european and northern white-breasted hedgehogs (Erinaceus europaeus and E. roumanicus) in Moscow region. Biology Bull 2009, 36, 647-651.

4. Caspersson T., Farbek S., Foley G.E., Kudynowski J., Modest E.J., Simonsson E., Wagh U., Zech T.: Chemical differentiation along metaphase chromosomes. Exp Cell Res 1969, 49, 219-222.

5. Gavrila L., Murariu D., Rebedea I., Mircea L., Stefan M., Vladimirescu A., Bucur S.: Preliminary results of the molecular cytogenetics study on hedgehog (Erinaceus concolor, Martin, 1838) (Mammalian: Insectivora) in Romania. Trav Mus Nat His Gr Antiopa 1998, 40, 431-448.

6. Geiser M., Gropp A.: Chromosome polymorphism in the European hedgehog, Erinaceus europaeus (Insectivora). Nature 1967, 214, 396-398.

7. Gropp A.: Cytologic mechanisms of karyotype evolution in insectivores. In: Comparative Mammalian Cytogenetics, edited by K. Benirschke, Springer, New York, 1969, pp. 247-266.

8. Howell W., Black D.A.: Controlled silver-staining of nucleolus organizer regions with a protective colloidal developer: a 1-step method. Experimentia 1980, 36, 1014-1015.

9. Hübner R., Maddalena T., Poduschka W.: The karyotype of the Middle-African hedgehog Atelerix albiventris Wagner, 1841 and its cytotaxonomical relationships to other Erinaceinae (Insectivora: Erinaceidae), Genetica 1991, 83, 243-246.

10. Král B.: Karyological analysis of two European species of the genus Erinaceus. Zoologické Listy 1967, 16, 239-252.

11. Levan A., Freda K., Sandberg A.A.: Nomenclature for centromeric position on chromosomes. Hereditas 1964, 52, 201-220.

12. Mandahl N.: Variation in C-stained chromosome regions in European hedgehogs (Insectivora, Mammalia), Hereditas 1978, 89, 107-128.

13. Mandahl N.: Localization of nucleolar organizing regions in European hedgehogs (Insectivora, Mammalia). Hereditas 1979, 91, 149-161.

14. Sánchez A., Jiménez R., Burgos M., Stitou S., Zurita F., Diaz de la Guardia R.: Cytogenetic peculiarities in the Algerian hedgehog: silver stains not only NORs but also heterochromatic blocks, Heredity 1995, 75, 10-16.

15. Schweizer D., Ambros P.F.: Chromosome banding stain combinations for specific regions. Method Mol Biol 1994, 29, 97-112. 